\title{
Evaluación de un programa psicoterapéutico para cuidadores de pacientes con TCA: Estudio piloto
}

\author{
Vanessa Pla \\ Eugenia Moreno \\ (Clínica Trastornos Alimentarios, Valencia, España) \\ Inmaculada Fuentes-Durá \\ Carmen Dasí \\ (Universitat de València, Valencia, España)
}

\begin{abstract}
IPSTIMPI/AhSTRE":
El objetivo de esta investigación es llevar a cabo un estudio piloto para evaluar un programa psicoterapéutico para cuidadores de pacientes diagnosticados de trastorno de la conducta alimentaria (TCA). El programa se ha aplicado a 13 cuidadores, todos ellos familiares de los pacientes, y está compuesto por 12 sesiones agrupadas en tres bloques. El estudio se desarrolla a lo largo de un año entero, ya que la evaluación de los cuidadores y pacientes se realiza antes de la intervención, al finalizar ésta y en una evaluación de seguimiento a los 6 meses. Los resultados de los familiares han mostrado una mejoría significativa en la experiencia como cuidador, una menor acomodación y mayor competencia frente al TCA, aumento de la satisfacción familiar, así como una disminución de los niveles de ansiedad y depresión. En los pacientes también se observó una disminución significativa de la sintomatología del trastorno.
\end{abstract}

The objective of this research is to conduct a pilot study to evaluate a psychotherapeutic program for caregivers of patients diagnosed with eating behavior disorder. The program has been applied to 13 caregivers, all relatives of patients, and is composed of 12 sessions grouped in three blocks. The study is developed over a whole year, because the assessment of caregivers and patients is performed before intervention, at the end of this and follow-up evaluation at 6 months. The results of the caregivers have shown a significant improvement in the experience as a caregiver, less accommodation and more competition against TCA, increased family satisfaction, as well as decreased levels of anxiety and depression. In patients also showed a significant decrease of symptoms of the disorder.

\section{palaloras clave/keywords:}

Trastorno conducta alimentaria (TCA), programa psicoterapéutico cuidadores, salud mental en familiares cuidadores.

Eating disorders (ED), caregivers psychotherapeutic program, mental health in family caregivers.

En los últimos años la incidencia de la anorexia nerviosa (AN), bulimia nerviosa (BN), y otras patologías afines, como el trastorno de la conducta alimentaria no específico (TCANE) ha aumentado considerablemente, convirtiéndose en un importante problema socio-sanitario que cada vez afecta a más adolescentes y en edades más tempranas. Diferentes estudios epidemiológicos efectuados en población española señalan que entre un $2 \%$ y un $4 \%$ de mujeres jóvenes padecen TCA. En adolescentes españolas con edades comprendidas entre los 12 y los 18 años, la prevalencia 
del trastorno se sitúa entre un 3,3\% y un $5,5 \%$ (Rojo et al., 2003), mientras que según el DSM-IV-TR (APA, 2002) es del $0,5 \%$. Sin embargo si atendemos a entidades subclínicas y síndromes parciales de la enfermedad, estas tasas se incrementan preocupantemente en mujeres, hasta valores próximos al $15-18 \%$ de la población juvenil. Esto ha provocado gran preocupación social que se ha manifestado en que la investigación de los TCA haya aumentado mucho en las últimas décadas. Entre las variables más estudiadas se encuentran la influencia social, los factores de personalidad individuales que intervienen en la génesis de estos trastornos y la influencia de la familia, sobre todo a través del tipo de interacciones que tienen lugar en el seno de estas familias. Prueba de ello son numerosas investigaciones en los últimos años dirigidas de manera específica a los cuidadores de TCA, como por ejemplo: Coomber y King (2012); Goddard, Macdonald y Treasure (2011); González, Padierna, Martín, Aguirre y Quintana (2012); Sepúlveda, Todd, Whithaker, Grover, Stahl y Treasure (2010).

Ochoa de Alda, Espina y Ortego (2006) han señalado que los padres de hijos con TCA presentan más trastornos afectivos y mayor neuroticismo que los controles, planteándose la posible etiología de la patología de las hijas. Por otra parte, otros autores han señalado lo contrario (Kendrick, 1999; Maurin y Boyd, 1990), no se trata de rasgos propios en sí del cuidador, sino que la patología de los hijos puede ser la causa de las alteraciones afectivas de los padres, resaltando la carga que representa para la familia tener un miembro con una enfermedad psiquiátrica. Kendrick (1999) y Maurin y Boyd (1990) afirman que esta alteración en los cuidadores sería consecuencia del cambio en la vida familiar, el aislamiento social y las dificultades económicas que la situación implica, llevando a los familiares a experimentar reacciones psicopatológicas como depresión, ansiedad y sentimientos de pérdida frente a la nueva situación.

En algunos estudios como Espina y Fernández (1996) y Espina, Pumar, González, Santos, García y Ayerbe (2000), se analizó el porcentaje de cuidadores que presentaban niveles clínicos de ansiedad obteniendo un $37,1 \%$, cantidad claramente superior a la obtenida en cuidadores de hijos con diagnóstico de esquizofrenia $(15 \%)$ y en cuidadores de hijos con toxicomanías $(19,1 \%)$. Los autores atribuyen estos resultados al estrés que ocasionan los TCA en la persona que se hace más cargo del paciente, generalmente la madre.

En ambas investigaciones se estudió también la sintomatología depresiva, encontrando porcentajes algo mayores en las madres cuidadoras de las otras psicopatologías respecto a los cuidadores de TCA (75\% toxicomanía, 70\% esquizofrenia y 63,33\% en TCA). Martín, Padierna, Aguirre, Quintana, Hayas y Muñoz (2011) concluyen que tanto las madres como los padres de pacientes con TCA presentan puntuaciones más bajas en salud mental, necesitando por lo tanto una atención especializada. Sepúlveda et al. (2012) también concluyen que trabajando las relaciones familiares se produce un descenso en la ansiedad y depresión de los cuidadores.

Todos estos estudios han demostrado la importancia que la salud mental de los cuidadores tiene sobre la evolución del TCA de los pacientes y por lo tanto la necesidad de hacer intervenciones específicas para ellos. Vandereycken, Castro y Vanderlinden 
(1991) desarrollaron en Bélgica grupos de apoyo para padres y madres de hijas diagnosticadas de TCA. El objetivo de estos grupos, más que alcanzar una meta psicoterapéutica particular, era aconsejar y apoyar a los familiares, combinando elementos psicoeducativos y de toma de conciencia.

A partir del programa de Anderson, Reiss y Hogarty (1986) para cuidadores de pacientes con esquizofrenia, Salorio, Ruiz, Torres, Sanchis y Navarro (1999) realizaron en España una versión para temas específicos de la alimentación, obteniendo resultados altamente satisfactorios.

Dare y Eisler (2000), Scholz y Asen (2001) y Tantillo $(2003,2006)$ llevaron a cabo programas psicoeducativos en un contexto de terapia multifamiliar, encontrando resultados satisfactorios, tanto para los familiares como para los pacientes. Esta modalidad de aplicación permite atender a mayor número de familias manteniendo la eficacia en sus intervenciones.

Entre los principales investigadores en terapia familiar cabe destacar a Janet Treasure. Su grupo de trabajo desarrolló en los 80 un modelo de intervención conocido como modelo Maudsley por el hospital de Londres en el que fue llevado a cabo. Sus trabajos (Treasure et al., 2008a, 2008b) indican que ciertos patrones de interacción de las familias pueden actuar como factores mantenedores de la enfermedad. A medida que el TCA va asentándose, la percepción de autoeficacia de los padres va mermando, viendo más complejo el proceso de recuperación y afectando a la salud mental de todos los miembros de la familia.

Schmidt y Treasure (2006) apuntan que la transformación que experimenta la vida familiar es una demostración directa de las relaciones disfuncionales que se producen en el seno de estas familias, entendiendo estas relaciones más como un factor causal de la enfermedad, que consecuencia de ésta en sí. Por lo tanto, entender cómo la familia reorganiza su vida alrededor de la sintomatología enferma, es fundamental para un tratamiento eficaz. Se considera importante que la familia identifique y esté preparada para hacer los cambios necesarios en las relaciones familiares, tomando consciencia del papel activo que pueden tener en la resolución de la sintomatología enferma, con el objetivo de que la situación tenga menores consecuencias en su salud mental. Diferentes análisis estadísticos han comprobado la eficacia del modelo, siendo sus ventajas mantenidas cinco años después (Goddard et al., 2011). Concretamente, el modelo es más eficaz cuantos menos años de evolución presenta la enfermedad.

Con el objetivo de continuar profundizando en la búsqueda de terapias familiares efectivas para ayudar al tratamiento de los TCA, se ha diseñado un programa de intervención siguiendo algunas de las pautas de los diferentes enfoques anteriormente nombrados. Por una parte, siguiendo los programas psicoeducativos de Anderson et al. (1986), se les proporciona a las familias información respecto a qué es un TCA, su génesis, evolución y sintomatología. Por otra parte, se pretende conseguir en las familias los objetivos marcados por el modelo Maudsley, como es el enseñar a los familiares habilidades de comunicación y de negociación frente a la sintomatología enferma para conseguir un afrontamiento más adaptativo a esta sintomatología.

Además, siguiendo los criterios del INSALUD (2008), con el objetivo de prevenir recaídas, se añadió una sesión al final 
del programa para que los familiares tengan información respecto a cómo actuar de manera eficaz al aparecer los primeros síntomas. Los objetivos particulares del programa objeto de este análisis son: a) conseguir una mejoría en la experiencia personal como cuidador, en general, y más específicamente en la experiencia como cuidador de TCA; b) disminuir el grado de acomodación y aumentar la competencia frente a la sintomatología alimentaria; c) tener una mayor satisfacción con la situación familiar general al trabajar la familia unida como un equipo; y d) a medida que los familiares cambian sus patrones de interacción con la enfermedad del hijo, cabe suponer que la sintomatología de TCA verá reducidos sus niveles. Respecto a las variables de salud mental: disminuir los niveles de ansiedad y depresión, al sentirse el cuidador más capaz de afrontar las situaciones generadas por el TCA.

\section{Método}

\section{Participantes}

El programa se implementó en una clínica de Valencia (España) especializada en el diagnóstico, tratamiento multidisciplinar y seguimiento de los TCA. Siguiendo los protocolos terapéuticos (INSALUD, 2008), los familiares de los pacientes acuden a una terapia familiar grupal para ayudarles a hacer frente a la situación generada por el TCA. En dichas sesiones es donde se llevó a cabo el programa cuya eficacia se quiere contrastar.

\section{Muestra de cuidadores.}

La muestra estuvo formada por trece padres cuidadores de once pacientes diagnosticados de TCA que recibían tratamiento en la clínica. El rango de edad iba de los 41 a los 59 años, $\mathrm{M}=49,0$ años (DT=5,6). Todos convivían de forma continuada con los pacientes. Tres de ellos eran padres $(23,1 \%)$ y diez madres (76,9\%). Respecto al estado civil, todos los cuidadores estaban casados, a excepción de una madre que era viuda. Se invitó a participar en la terapia a ambos miembros de la pareja, pero por distintas razones sólo en dos pacientes participaron tanto el padre como la madre. En la Tabla 1 se describen otras características relevantes de los familiares.

\section{Muestra de pacientes.}

Los familiares participantes eran cuidadores habituales del grupo de once pacientes, todos ellos habían sido previamente diagnosticados con TCA según el DSM-IV-TR (2002) en la clínica especializada en TCA donde se desarrolló la investigación. Res-

Tabla 1. Principales variables descriptivas de los cuidadores.

\begin{tabular}{lllll}
\hline Estudios realizados $(\%)$ & Dedicación laboral $(\%)$ & $\begin{array}{l}\text { Horas contacto } \\
\text { semanal }(\%)\end{array}$ & $\begin{array}{l}\text { Terapias } \\
\text { anteriores }(\%)\end{array}$ & $\begin{array}{l}\text { Antecedentes } \\
\text { familiares TCA }\end{array}$ \\
\hline Superiores $(46,1)$ & Jornada completa $(53,8)$ & $+41(53,8)$ & No $(76,9)$ & No $(69,2)$ \\
Secundarios $(23,1)$ & Media jornada $(23,1)$ & $31-40(0)$ & Sí $(23,1)$ & $\left(^{*}\right)$ Sí $(30,8)$ \\
Primarios $(30,8)$ & Amas casa $(23,1)$ & $21-30(15,4)$ & & \\
& & $11-20(23,1)$ & & \\
& & $0-10(7,7)$ & & \\
\hline
\end{tabular}

$\left({ }^{*}\right)$ Familiar de primer grado 
pecto al género de los pacientes, nueve eran chicas $(85 \%)$ y dos chicos $(15 \%)$. El rango de edad iba de los 13 a los 29 años, siendo la media 18,5 $(\mathrm{DT}=5,4)$. Respecto al tiempo de evolución del trastorno, variaba desde 2 hasta 48 meses, $M=23,9$ meses (DT $=17,3$ meses). En relación al tiempo que llevan recibiendo tratamiento, también varía desde una semana a 48 meses, $M=9,7$ meses (DT=13,6 meses). En la Tabla 2 se describen otras características relevantes de los pacientes. sin acomodarse a sus síntomas y siguiendo los principios de la terapia motivacional de Miller y Rolnick (1993). En el bloque II (sesiones 4, 5, 6, 7, 8, 9, 10 y 11) se trabajan las reacciones más frecuentes del cuidador de TCA, con el objetivo de que aprendan a llevar a cabo una respuesta más eficaz. Se trabajan las habilidades de comunicación, pretendiendo un cambio positivo en el lenguaje familiar y nociones básicas acerca del análisis funcional de conducta. El bloque III (sesión 12) es sobre prevención de recaídas. Se les explica a los fami-

Tabla 2. Principales variables descriptivas de

los pacientes.

\begin{tabular}{llll}
\hline Estudios en curso (\%) & Diagnóstico clínico (\%) & $\begin{array}{l}\text { Modalidad tratamiento } \\
\text { inicio programa(\%) }\end{array}$ & $\begin{array}{l}\text { Modalidad tratamiento final } \\
\text { programa(\%) }\end{array}$ \\
\hline $\begin{array}{l}\text { Universidad }(36,3) \\
\text { Bachillerato }(27,3)\end{array}$ & AN restrictiva $(54,5)$ & Hospital día $(100)$ & Hospital día $(27,3)$ \\
$\begin{array}{l}\text { Secundaria }(27,3) \\
\text { Primaria }(9,1)\end{array}$ & TCANE $(9,1)$ & Ambulatorio $(0)$ & Ambulatorio $(72,7)$ \\
\hline
\end{tabular}

\section{Procedimiento}

Los cuidadores fueron informados acerca de la voluntariedad de la realización del programa y todos ellos firmaron el consentimiento. El programa constaba de 3 bloques, con un total de 12 sesiones de hora y media. En el bloque I de psicoeducación (sesiones 1, 2 y 3) se les explica a los familiares que es el TCA, las características que los definen y los factores multicausales que los generan. Se les enseñan las etapas de cambio por las que van pasando tanto el paciente como los familiares durante el proceso de enfermedad. El objetivo es que los familiares puedan identificar la sintomatología enferma para que puedan practicar las habilidades de negociación frente a ésta, liares en qué consiste una recaída, cómo poderla prevenir y cuáles son las cuestiones principales a tener en cuenta para poder paliar sus consecuencias.

Cada sesión se estructuraba de la siguiente manera: durante los 10 primeros minutos se realizaban ejercicios de relajación, autoestima, mindfulness, lectura de cuentos y ejercicios prácticos, para que los padres aprendieran a tomar conciencia de su estado emocional, de sus habilidades de resolución de problemas y reflexionasen sobre algunas cuestiones importantes que después iban a ser trabajadas en la sesión; a continuación, durante la hora y veinte minutos restante, se llevaba a cabo la parte psicoeducativa y 
de práctica de las habilidades y estrategias psicoterapéuticas objetivo de dicha sesión. La periodicidad era cada 2 semanas, excepto si coincidía con día festivo en cuyo caso el intervalo intersesiones era de 3 semanas. La duración total fue de 6 meses.

Antes de iniciarse las sesiones se midieron las principales variables relacionadas con los TCA, tanto a los familiares como a los pacientes. En la Tabla 3 aparecen dichas variables así como los instrumentos de medición utilizados. Cuando finalizó el programa se volvieron a pasar las pruebas para medir de nuevo las variables dependientes y 6 meses después se volvió a realizar una medición de seguimiento, por lo que la investigación se desarrollo a lo largo de un año. Según la clasificación de Montero y León (2007) es una investigación cuasiexperimental de un grupo con medidas pre-post tratamiento y seguimiento a los 6 meses.

\section{Instrumentos empleados en la evaluación de los cuidadores:}

AESED - Escala de acomodación e incompetencia a los síntomas alimentarios (Sepúlveda, Kyriacou y Treasure, 2009). Evalúa mediante 47 ítems en qué medida los cuidadores organizan sus interacciones diarias con la sintomatología del TCA. Está compuesta por cinco factores: evitación y modificación de rutinas, conductas de comprobación, rituales alimentarios, control de la familia y punto ciego. Su alfa de Cronbach está situado entre 0,77 y 0,92 según el factor.

BDI - Beck Depression Inventory (Beck, Ward, Mendelson, Mock y Erbaugh, 1961). Cuestionario autoaplicado formado por 21 ítems de respuesta múltiple relacionados con síntomas depresivos, como la desesperanza e irritabilidad, cogniciones como culpa, o sentimientos como estar siendo castigado, así como síntomas físicos relacionados con la depresión. La fiabilidad es de 0,90 y los índices de la validez de criterio adecuados.

ECI - Inventario de la experiencia del cuidador (Szmukler, Burgess, Herrman y Benson, 1996). Cuestionario autoaplicado compuesto por 66 ítems que evalúa el estrés, la percepción y el afrontamiento de la situación de los cuidadores de personas con enfermedad mental severa. Está compuesto por diez escalas. Ocho de ellas miden los aspectos negativos del cuidado, la llamada dimensión negativa: comportamientos difíciles, síntomas negativos, estigma, problemas con los servicios, efectos en la familia, necesidad de apoyo, dependencia y pérdida. Mayor puntuación indica una experiencia más negativa del cuidador. Las otras dos escalas miden la dimensión positiva: experiencias personales positivas y aspectos buenos de la relación con el paciente. El alfa de Cronbach es muy elevada $(0,95)$. En esta investigación interesa sobre todo la dimensión negativa.

EDSIS - Impacto de síntomas del trastorno alimentario (Sepúlveda, Whitney, Hankins y Treasure, 2008). Para la evaluación de la carga específica del cuidador de TCA, a la escala ECI se le ha añadido 24 ítems más, referidos a la experiencia específica como cuidador de una persona con TCA. Esta escala está diseñada para medir el impacto personal que los síntomas del TCA y el comportamiento del familiar enfermo tienen en el bienestar del cuidador en el último mes. Consta de 4 factores, dos de los cuales están directamente relacionados con los problemas específicos causados por los síntomas del TCA: impacto de los aspec- 
tos nutricionales y alteración conductual, mientras que los otros dos están relacionados con las reacciones personales del cuidador frente a la enfermedad: culpa y aislamiento social. Las propiedades psicométricas son adecuadas ya que su fiabilidad es de 0,92 .

\section{ESFA - Escala de satisfacción familiar por adjetivos (Barraca y López-Yarto,} 1997). Escala unidimensional diseñada para dar una medida global de la satisfacción familiar. Mediante 27 adjetivos trata de evocar respuestas, preferentemente afectivas, para medir los sentimientos positivos o negativos que se despiertan en el cuidador en las interacciones con los miembros de la familia. Las propiedades psicométricas de la escala son buenas, siendo el coeficiente de fiabilidad es de 0,87 .

\section{STAI - Cuestionario de ansiedad Estado/} Rasgo (Spielberger, Gorsuch y Lushene, 1980). La prueba consta de dos partes, con 20 cuestiones en cada una de ellas. La primera, ansiedad estado (A/E) evalúa un estado general transitorio, caracterizado por sentimientos subjetivos, conscientemente percibidos, de atención y aprensión por hiperactividad del sistema nervioso autónomo. La segunda, ansiedad rasgo (A/R) señala una propensión ansiosa, relativamente estable, que caracteriza a los individuos con tendencia a percibir las situaciones como amenazadoras. El STAI posee una buena consistencia interna (entre 0,90 y 0,93 en $\mathrm{A} / \mathrm{E}$ y entre 0,84 y 0,87 en $\mathrm{A} / \mathrm{R}$ ).

Autoinforme consistente en un ítem que se contestaba en cada sesión del programa para medir el bienestar del cuidador en una escala desde máximo malestar (0) a máximo bienestar (10).
Autoinforme consistente en un ítem que se contestaba en cada sesión del programa para medir la percepción del cuidador de la gravedad de la patología del hijo/a en una escala de 10 puntos desde máxima gravedad (0) a salud óptima (10).

\section{Instrumentos utilizados para la evaluación de los pacientes:}

EAT 40 - Eating Attittude Test (Garner y Garfinkel, 1979). Su objetivo es medir de forma general los síntomas del TCA. Es una prueba autoaplicada compuesta de 40 ítems agrupados en siete factores: conductas bulímicas, imagen corporal con tendencia a la delgadez, abuso de laxantes, presencia de vómitos, restricción alimentaria, comer a escondidas y presión social percibida al aumentar de peso. El índice de consistencia interna es de 0,94.

EDI 2 - Inventario de trastornos de la conducta alimentaria (Garner, 1991). El EDI contiene 64 reactivos agrupados en ocho subescalas: obsesión por la delgadez, bulimia, insatisfacción corporal, ineficacia, perfeccionismo, desconfianza interpersonal, conciencia interoceptiva y miedo a la madurez. En el EDI-2 se introducen 27 reactivos adicionales clasificados en otras tres subescalas: ascetismo, impulsividad e inseguridad social. Presenta un índice de fiabilidad de 0,95 .

\section{Resultados}

Los análisis se realizaron con el paquete estadístico IBM SPSS Statistics 19. En la Tabla 3 se resumen los principales indicadores de la evolución de las variables dependientes medidas antes (preprograma), después (postprograma) y a los 6 meses (seguimiento), tanto en la muestra de pacientes como en la de sus cuidadores. Los 
contrastes de medias para todos los tests se realizaron con ANOVAS unifactoriales de medidas repetidas. Todos los supuestos de dicho ANOVA fueron comprobados. Sólo en 3 de los contrastes efectuados no se cumplía el supuesto de esfericidad por lo que se utilizó el epsilon de GreenhouseGeisser para corregir los grados de libertad (Tabla 3).

\section{Análisis estadísticos de los datos de los cuidadores}

En la Tabla 3 se aprecia un descenso progresivo en las medias de la puntuación total del AESED, lo que indica que después del programa los cuidadores presentaban una mayor competencia en el afrontamiento del TCA y una menor acomodación a los síntomas alimentarios de los hijos. La prueba de comparaciones múltiples con el ajuste de Bonferroni fue significativa al hacer la comparación pre-post $(\mathrm{p}<0,01)$ y pre-seg $(p<0,001)$, pero no lo fue en el contraste post-seguimiento.

Por lo que se refiere al BDI la media preprograma indicaba un nivel bajo de sintomatología depresiva previo al programa. La evolución de las medias fue muy satisfactoria, tal como se aprecia en la Tabla 3. El ajuste de Bonferroni mostró la presencia de diferencias significativas al comparar las medias pre-seguimiento $(\mathrm{p}<0,05)$.

Es importante destacar que algunos cuidadores tenían puntuaciones suficientemente altas para un diagnóstico de depresión moderada, y fueron precisamente ellos los que al finalizar el programa vieron reducidos sus niveles hasta valores indicativos de ausencia de sintomatología depresiva. Es decir, aquellos cuidadores que presentaban niveles de depresión más altos, son también los que redujeron de forma más notable su sintomatología. Este resultado cumple los criterios de significación clínica del método de comparación con muestras normativas (Kazdin, 2001), a saber, nadie presentaba niveles clínicos de depresión después del programa.

Los análisis del ECI se han centrado en la dimensión negativa que es la más importante para los objetivos del programa. Según la prueba de Bonferroni, después de la intervención los familiares presentaban un descenso significativo respecto al principio $(\mathrm{p}<0,05)$. Por lo tanto, los cuidadores presentaban una mejoría en la experiencia personal frente al estrés, a la percepción de la enfermedad de su familiar y respecto al afrontamiento de la sintomatología. La comparación pre-seguimiento también fue significativa $(\mathrm{p}<0,001)$ lo que indica que los cambios se mantuvieron en el tiempo.

En el EDSIS se volvió a repetir el mismo patrón de resultados ya que se obtuvieron diferencias significativas tanto en la comparación pre-post $(\mathrm{p}<0,05)$, como en la comparación pre-seguimiento $(\mathrm{p}<0,001)$. Estos datos indican una disminución relevante del impacto personal que los síntomas del TCA tiene sobre los cuidadores.

En la escala ESFA, la media inicial de 46,15 puntos indica que los cuidadores tenían una satisfacción familiar ligeramente inferior a la media de la población general. Sin embargo, tal como se aprecia en la Tabla 3 , después del programa había subido hasta 70,38 puntos. Dicha diferencia fue significativa $(p<0,005)$ según el ajuste de Bonferroni y mantenida en el tiempo, ya que no hubo diferencias significativas entre postprograma y seguimiento.

En relación a los datos de la escala STAI, la evolución fue satisfactoria, tanto para la 
ansiedad estado como rasgo, encontrando una disminución significativa al comparar la puntuación centil media preprograma y la del seguimiento $(p<0,05)$. Probablemente debido a la gran variabilidad de las puntuaciones y lo escaso de la muestra no se alcanzó la significación al comparar las medias pre y postprograma a pesar de que el descenso en la ansiedad era de más de 10 puntos.

Dado que en este estudio la muestra es reducida es muy importante realizar algún cálculo que nos permita conocer la magnitud de las diferencias observadas con independencia del tamaño muestral, esto es, una medida del tamaño del efecto como $\eta^{2}$ parcial, que es una medida de la proporción de la varianza explicada. En la última columna de la Tabla 3 se observa este indicador. Considerando los criterios de Cohen (1988) se puede afirmar que en todos los contrastes efectuados con los datos de los cuidadores el tamaño del efecto ha sido grande.

Por lo que se refiere a los autoinformes sobre bienestar del cuidador y salud del paciente obtenidos en las 12 sesiones, se ha obtenido una correlación lineal de 0,815 $(\mathrm{p}<0,005)$ indicando que mayor bienestar

Figura 1: Correlación entre el bienestar del cuidador y la salud de la hija.

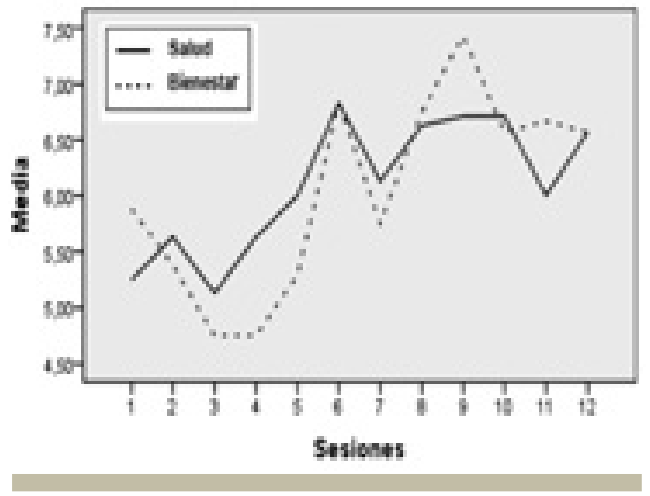

Tabla 3. Resultados de la evaluación de los cuidadores y los pacientes previa al programa, al acabar el programa y en el seguimiento a los 6 meses.

\begin{tabular}{ccccccc}
\hline Cuidadores & \multicolumn{7}{c}{} & & & \\
\hline Prueba & $\begin{array}{c}\text { Preprograma } \\
\mathrm{M}(\mathrm{DT})\end{array}$ & $\begin{array}{c}\text { Postprograma } \\
\mathrm{M}(\mathrm{DT})\end{array}$ & $\begin{array}{c}\text { Seguimiento } \\
\mathrm{M}(\mathrm{DT})\end{array}$ & ANOVA & $\begin{array}{c}\mathrm{h} 2 \\
\text { parcial }\end{array}$ \\
\hline AESED & $40,1(16,8)$ & $20,7(12,2)$ & $15,8(11,7)$ & $\mathrm{F}(2 ; 24)=15,3$ & 0,001 & 0,56 \\
\hline BDI & $10,1(7,5)$ & $7,1(6,3)$ & $3,9(3,7)$ & $F(2 ; 24)=6,5$ & 0,005 & 0,35 \\
\hline ECI Negativo & $65,2(21,0)$ & $39,2(23,7)$ & $33,3(17,6)$ & $F(1,2 ; 14,6)=16,8$ & 0,001 & 0,58 \\
\hline EDSIS & $27,9(11,4)$ & $17,2(14,2)$ & $12,5(10,3)$ & $F(2 ; 24)=11,4$ & 0,001 & 0,49 \\
\hline ESFA & $46,2(24,7)$ & $70,4(19,6)$ & $73,9(22,2)$ & $F(2 ; 24)=15,9$ & 0,001 & 0,57 \\
\hline STAI-E & $62,3(26,8)$ & $47,3(30,2)$ & $36,3(35,3)$ & $F(2 ; 24)=4,4$ & 0,05 & 0,27 \\
\hline STAI-R & $59,8(24,7)$ & $49,7(27,0)$ & $34,6(26,6)$ & $F(2 ; 24)=8,5$ & 0,005 & 0,42 \\
\hline Pacientes & & & & & & \\
\hline EAT-40 & $28,2(18,9)$ & $6,9(6,3)$ & $7,6(4,7)$ & $F(1,1 ; 11,0)=15,0$ & 0,005 & 0,60 \\
\hline EDI-2 & $70,6(52,0)$ & $43,1(29,7)$ & $26,9(30,9)$ & $F(1,1 ; 11,0)=5,2$ & 0,05 & 0,34 \\
\hline & & & & &
\end{tabular}


en el cuidador va asociado a mayor salud del paciente (Figura 1).

La tendencia en ambas variables es claramente ascendente, ya que a medida que se van desarrollando las sesiones, se va produciendo un aumento tanto del bienestar del cuidador y como de la salud del paciente a su cargo, apareciendo una correlación lineal significativa entre sesiones y bienestar $\mathrm{R}=0,70(\mathrm{p}<0,05)$, así como entre sesiones y salud $R=0,75(p<0,01)$.

\section{Análisis estadísticos de los datos de los pacientes}

En el test EAT-40, la puntuación media obtenida por los hijos al inicio de la intervención se redujo en 20 puntos en la medición postprograma, por tanto, hubo un descenso general de los síntomas del TCA. El test de Bonferroni indicó que la diferencia entre el pre y el postprograma fue significativa $(p<0,01)$ y no lo fue entre el postprograma y el seguimiento, o sea, se mantuvo el descenso.

En el EDI-2 hubo una disminución de casi 30 puntos entre la medición pre y postprograma, que alcanzó también la significación estadística $(p<0,01)$, lo que indica un gran descenso en insatisfacción, ineficacia, perfeccionismo, desconfianza interpersonal, etc. Los cambios se mantuvieron ya que no hubo diferencias entre el postprograma y el seguimiento.

Los cálculos del tamaño del efecto $\left(\eta^{2}\right.$ parcial) mostraron que también con los datos de los cuidadores los tamaños del efecto obtenidos son grandes (Cohen, 1988). Tal como predice el modelo Maudsley, las intervenciones más eficaces serán en aquellos pacientes en los que el periodo de evolución de la enfermedad sea menor y antes se intervenga para paliar sus síntomas $(\mathrm{r}=-0,62, \mathrm{p}<0,05)$. Es decir, resulta una correlación negativa entre el tiempo de evolución de la enfermedad y la mejoría de los hijos.

Todos los análisis que han sido descritos en este apartado se hicieron también teniendo en cuenta el tipo de diagnóstico del paciente (AN y TCANE), no apareciendo en ninguna variable dependiente diferencias significativas entre ambos grupos, por lo que se ha realizado la descripción de los análisis sobre dichas variables sin hacer referencia al diagnóstico clínico.

\section{Discusión y conclusiones.}

Los resultados obtenidos permiten ser optimistas respecto a la utilidad del programa de intervención familiar utilizado, ya que parece ayudar bastante a los padres a mejorar su experiencia personal como cuidadores, y están en la línea de lo que apuntaban Eisler et al. (1997) respecto a que la intervención en los familiares de pacientes jóvenes y con una duración corta del TCA es efectiva para paliar los síntomas del trastorno.

La mejoría que presentan los familiares después de la intervención en la puntuación del ECI, indica una reducción del estrés, una percepción más adaptativa de la enfermedad, un mejor afrontamiento de su sintomatología, una reducción del grado en que sienten que la enfermedad les estigmatiza, una disminución de la dependencia del familiar y una mejoría en el sentimiento de pérdida.

Los resultados del EDSIS indican un aumento en el bienestar del cuidador en lo que respecta a la carga específica que supone el cuidado de su hijo enfermo. Los cuidadores presentaban un menor impacto en los fac- 
tores que están directamente relacionados con problemas específicos causados por los síntomas del TCA (impacto de los aspectos nutricionales y alteración conductual), así como en las reacciones personales del cuidador frente a la enfermedad (culpa y aislamiento social). Se considera muy importante esta reducción del sentimiento de culpa. La información psicoeducativa proporcionada en el Bloque I del programa ha ayudado a rebajar los niveles de malestar personal, permitiéndoles un afrontamiento activo en la resolución del trastorno. Así también, los cuidadores han ido aprendiendo más recursos para no ceder a las situaciones que el TCA plantea, sin organizar la vida familiar alrededor del trastorno, cuentan con más estrategias de afrontamiento.

En referencia al nivel de satisfacción familiar evaluada en el ESFA, después de la intervención se observa un cambio positivo en los cuidadores. Éste era uno de los objetivos principales de la intervención, ya que se esperaba un cambio en la respuesta afectiva del cuidador hacia la familia, un aumento en los sentimientos positivos que se despiertan en ellos al interactuar en las situaciones familiares, facilitando de este modo respuestas más cálidas en el cuidador.

En relación a los niveles de depresión (BDI) de los cuidadores, ya desde el inicio de la intervención la mayoría no presentan niveles de depresión clínica. Es importante destacar que los cuidadores que mostraban al inicio de la intervención niveles patológicos de depresión moderada, al finalizar ésta, vieron reducidos sus niveles hasta valores indicativos de no depresión. Se consigue, por tanto, uno de los objetivos que se habían planteado al inicio, la disminución de la sintomatología depresiva.
Así también los resultados obtenidos en el STAI, tanto rasgo como estado, indican que los familiares después de la intervención han reducido la ansiedad de forma importante y tienden a percibir las situaciones como menos amenazantes.

La mejoría en la situación general del cuidador está acompañada de una mejoría en la situación clínica de los hijos. A medida que los cuidadores han ido cambiando sus patrones de interacción familiar y han ido aprendiendo a afrontar la sintomatología, colaboran como un agente activo en el proceso de recuperación. Se trata pues de un proceso circular ya que el bienestar del cuidador y la salud del paciente se están continuamente retroalimentando. En línea con el modelo Maudsley, las intervenciones familiares más eficaces serán en aquellos pacientes en los que el periodo de evolución de la enfermedad sea menor y antes se resuelva la disfunción familiar que mantiene el TCA.

Una importante limitación de esta investigación es la ausencia de grupo control, que permitiría evaluar los factores relacionados con la propia evolución del trastorno asociados al tiempo (maduración pacientes, desarrollo habilidades en cuidadores, etc...). Por razones de ética profesional no es lícito dejar sin intervención familiar a ningún paciente por tener grupo control. Otra de las limitaciones es el reducido tamaño de la muestra. Esto, aunque a priori puede parecer una gran desventaja no lo es tanto, ya que si con una muestra tan pequeña se ha alcanzado la significación estadística en la mayoría de los contrastes efectuados, cabe suponer que el aumento en la potencia que se conseguiría al aumentar el tamaño de la muestra podría redundar en que algunas diferencias que no han alcan- 
zado la significación estadística lo hubieran hecho. De hecho, se trata de un estudio piloto que se está continuando en la actualidad con nuevos pacientes cuyos datos hasta el momento van en la línea de reforzar las conclusiones a las que se ha llegado en el piloto.

Parece interesante para próximas investigaciones comprobar si estos resultados, obtenidos con cuidadores de pacientes con diagnóstico de AN-R, AN-P y TCANE, son extrapolables a otros diagnósticos clínicos de TCA como la BN. Estos nuevos datos pueden arrojar más luz sobre los aspectos a mejorar en futuras aplicaciones del programa.

\section{Referencias}

Anderson, C.M., Reiss, D.J. y Hogarty, G.E. (1986). Schizophrenia and the Family: A Practitioner's Guide to Psychoeducation and Management. Buenos Aires: Amorrortu.

American Psychiatric Association, APA (2002). DSM-IVTR. Manual diagnóstico y estadístico de los trastornos mentales. Texto revisado. Barcelona: Masson.

Barraca, J. y López-Yarto, L. (1997). Escala de Satisfacción Familiar por Adjetivos. Madrid: TEA.

Beck, A.T., Ward, C. H., Mendelson, M., Mock, J. y Erbaugh, J. (1961) An inventory for measuring depression. Archives of General Psychiatry 4, 561-571.

Cohen, J. (1988). Statistical power analysis for the behavioral sciences (2 ${ }^{a}$ Ed.) Hillsdale, $\mathrm{NJ}$ : Lawrence Erlbaum.

Coomber, K. y King, R.M. (2012). Coping strategies and social support as predictors and mediators of eating disorder carer burden and psychological distress. Social Psychiatry Epidemiology, 47, 789-796.

Dare, C. y Eisler, I. (2000). A multyfamily group day treatment programme for adolescent eating disorders. European eatind disorders Review,8, 4-18.

Eisler, I., Dare, C., Russell, G.F., Szmukler, G., le Grange, D. y Dodge, E. (1997). Family and individual therapy in anorexia nervosa. A 5-year follow-up. Archives of General Psychiatry, 54, 1025-1030.
Espina, A. y Fernández, C. (1996). Un estudio descriptivo sobre características familiares en una muestra de Proyecto Hombre. Revista Española de Drogodependencias, 21, 109-118.

Espina, A., Pumar, B., González, P., Santos, A., García, E. y Ayerbe, A. (2000). Emoción expresada y características de personalidad, psicopatológicas y de ajuste diádico en padres de esquizofrénicos. Psiquis, 21, 23-33.

Garner, D. (1991). The eating disorders inventory 2. Odessa, FL: Psychological Assessment Ressources.

Garner, D. y Garfinkel, P. (1979). The eating attitudes test: an index of symptoms of anorexia nervosa. Psychological Medecine, 9, 273-279.

Goddard, E., Macdonald, P. y Treasure, J. (2011). An examination of the impact of the Maudsley Collaborative Care skills training workshops on patients with anorexia nervosa: a qualitative study. European eating disorders review, 19, 150-161.

González, N.; Padierna, A.; Martín, J.; Aguirre, U. y Quintana, J.M. (2012). Predictors of change in percieved burden among caregivers of patients with eating disorders. Journal of affective disorders, 139, 273-282.

INSALUD (2008). Guías de Práctica Clínica en el Sistema Nacional de Salud. Madrid: Plan de Calidad para el Sistema Nacional de Salud del Ministerio de Sanidad y Consumo.

Kazdin, A.E. (2001). Métodos de investigación en psicología clínica. México: Pearson education.

Kendrick, T. (1999). Primary care options prevent mental illness. Annals of Medicine, 31, 359-363.

Martín, J., Padierna, A., Aguirre, U., Quintana, J.M., Las Hayas, C. y Muñoz, P. (2011). Quality of life among caregivers of patients with eating disorders. Quality of life research, 20, 1359-1369.

Maurin, J.T. y Boyd, C.B. (1990). Burden of mental illness on the family: A critical review. Archives Psychiatric Nursing, 4, 99-107.

Miller, W.R. y Rollnick, S. (1993) Motivational Interviewing. Preparing people to change addictive behaviours. New York: Guildford Press.

Montero, I. y León, 0. (2007). A guide for naming research studies in Psychology. International Journal of Clinical and Health Psychology, 7, 847-862.

Ochoa de Alda, I., Espina, A. y Ortego, A. (2006) Un estudio sobre personalidad, ansiedad y depresión en padres de pacientes con un trastorno alimentario. Clínica y salud, 17, 151-170.

Rojo, L., Conesa, L., García, A., Domínguez, A., Rodrigo, G., Sanjuán, L. y Vila, M. (2003). Epidemiology and risc factors of eating disorders: a to stages epidemiologic 
study in a Spain population aged $12-18$ years. International journal of eating disorders, 34, 281-291.

Salorio, P., Ruiz, M.E., Torres, A., Sanchis, F. y Navarro, L. (1999) Psicoeducación familiar en grupo en los trastornos de la alimentación. Aplicación de un programa. Revista electrónica de psiquiatría, 3, 1.

Schmidt, U. y Treasure, J. (2006). Anorexia nervosa: Valued and visible. A cognitive interpersonal mantenance model and its implications for research and practice. British journal of clinical psychology, 45, 343-366.

Scholz, M. y Asen, E. (2001). Multiple family therapy with eating disorders adolescents: Concepts and preliminary results. European ating disorders review, 9, 33-42.

Sepúlveda, A.R., Graell, M., Berbel, E., Anastasiadou, D., Botella, J., Carrobles, J. y Morande, G. (2012). Factors associated with emocional well-being in primary and secondary caregivers of patients with eating disorders. European eating disorders review, 20, 78-84.

Sepúlveda, A.R., Todd, G., Whitaker, W., Grover, M., Stahl, D. y Treasure, J. (2010). Expressed emotion in relatives of patients with eating disorders following skills training program. International journal of eating disorders, 43, 603-10.

Sepúlveda, A.R., Kyriacou, 0. y Treasure, J. (2009). Development and validation of the Accomodation and Enabling Scale for Eating Disorders (AESED) for caregivers in eating disorders. Health Services Reasearch, 9, 171.

Sepúlveda, A.R., Whitney, J., Hankins, M. y Treasure, J. (2008). Development and validation of an Eating Disorders Symptom Impact Scale (EDSIS) for carers of people with eating disorders. Health and quality of life outcomes, 6, 28-42.

Spielberger, C., Gorsuch, R. y Lushene, R. (1980). STAI. Manual for the State-Trait Anxiety Inventory. Palo Alto: Consulting Psychology Press.

Szmukler, G.I., Burgess, P., Herrman, H. y Benson, A. (1996). Caring for relatives with serious mental illness: the development of the experience of care giving inventory. Social Psychiatry and Psychiatric Epidemiology, 31, 137148.

Tantillo, M. (2003) Eating disorders multifamily therapy group: capitalizing on the healing power of relationship. Eating disorders today, 2, 12-13.

Tantillo, M. (2006). A relational approach to eating disorders multifamily therapy group: moving from difference and diconnection to mutual connection. Families, systems \& health, 24, 82-102.

Treasure, J., Sepúlveda, A., Macdonald, P., Whitaker, W., Lopez, C., Zabala, M., Kyriacou, O. y Todd, G. (2008a). The assessment of the family of people with eating disorders. European eating disorders review, 16 , 247-255.
Treasure, J., Sepúlveda, A.R., MacDonald, P., Whithaker, W., Lopez, C., Zabala, M., Kyriacou, O. y Todd, G. (2008b) Interpersonal manteining factors in eating disorders: Skills sharing interventions for carers. International jounal of child and adolescent health, 1, 331-338.

Vandereycken W., Castro, J. y Vanderlinden, J. (1991). Anorexia y Bulimia. La familia en su génesis y tratamiento. Barcelona: Martínez-Roca.

Fecha de recepción: 06/03/2013 Fecha de aceptación: 17/10/2013 